\title{
Il dibattito linguistico e letterario nella poesia satirico-burlesca del primo Cinquecento in Italia
}

\author{
Benedict BUONO \\ Universidade de Santiago de Compostela \\ ben.buono@usc.es
}

\begin{abstract}
RESUMEN
En este artículo, el autor analiza la opinión de algunos autores de poesía satírica y burlesca sobre el canon lingüístico de la época y sobre las diferentes influencias literarias en la época, como el petrarquismo. Se analizan autores como Ercole Bentivoglio o Pietro Nelli.
\end{abstract}

Palabras clave: literatura italiana, poesía satírico-burlesca, siglo XVI, questione della lingua

[Recibido, septiembre 2013; aprobado, diciembre 2013]

The linguistic and literary debate in Italian satirical and burlesque poetry in the early $16^{\text {th }}$ century

\begin{abstract}
In this article, the author analyzes the opinion of some authors of satirical and burlesque poetry on the linguistic canon of their time and the different literary influences, such as the Petrarchan poetry. Authorslike Pietro Nelli andErcole Bentivoglio are analyzed.
\end{abstract}

Keywords: Italian literature, satirical poetry, $16^{\text {th }}$ century, questione della lingua 


\section{Introduzione}

Che la storia della lingua italiana possa essere correttamente interpretata attraverso una disanima dei grandi modelli letterari medievali e della loro successiva codificazione grammaticale cinquecentesca è un fatto non solo assodato, ma che ha ormai acquisito, a tutti i livelli, i caratteri dello stereotipo culturale, saldamente fondato sull'equazione tra fiorentino trecentesco e lingua comune. Pur condividendo le critiche sollevate da alcuni studiosi in merito a questo fondamentale nodo teorico ${ }^{1}$, è indubbio che Pietro Bembo, «umanista e classicista, interprete fedele delle esigenze formali del primo Cinquecento» (Vitale 1996: 7), abbia propugnato nelle sue Prose della volgar lingua la supremazia del volgare fiorentino su tutte le parlate d'Italia, additando come canone d'autorità per la poesia, con l'esclusione quindi della lingua viva, Dante e Petrarca. Fu soprattutto quest'ultimo, è noto, a incarnare i caratteri di esemplare perfezione letteraria e linguistica ${ }^{2}$, in virtù del suo stile sempre dominato da una sostanziale e lucida medietà, capace di dissolvere perfino gli inserti espressionistici di stile aspro mutuati dai suoi predecessori in un' «ineffabile tensione musicale del verso» (Vitale 1996: 14). Sul versante opposto, si trovava invece l'altro nume tutelare dell'italiano letterario, Dante, appunto, il cui plurilinguismo, sapientemente articolato su un ampio ventaglio di scelte lessicali - molte delle quali furono apostrofate dal Bembo come «rozze disonorate» e «non senza biasimo» (Prose, II, 5), dai latinismi ai forestierismi, dagli arcaismi ai neologismi e agli idiotismi -, poco si confaceva agli ideali classici e antidemotici del cardinale veneziano. Del resto, il dettato bembiano, espressione più autorevole della moda del fiorentinismo arcaizzante primo-cinquecentesco, seppe plasmare ad uso collettivo le variegate e multiformi esperienze individuali degli scrupolosi «osservatori» - soprattutto settentrionali $-{ }^{3}$ dei testi canonici del classicismo volgare: come afferma Dionisotti «Non vi fu letterato forse, che non usasse trasferire nei margini di un Petrarca vocaboli e costrutti del testo, collegando con rinvii da carta a carta quelli ripetuti o simili [...]. Dai margini, vocaboli e costrutti passavano poi, pari pari, in un sonetto o in un'altra composizione del postillatore» (Trovato 1994: 82). Tuttavia l'intento normativo bembiano era il risultato dell'elaborazione del nuovo ideale umanistico dell'epistola De imitatione, sorta dalla disputa originatasi intorno all'osservanza dei classici, che coinvolse Bembo e Gianfrancesco Pico della Mirandola tra il 1512 e il 1513 , vale a dire, negli stessi anni in cui vennero redatti i primi due libri delle Prose $e^{4}$. A

\footnotetext{
${ }^{1}$ Sulle riserve all'apporto di Dante e Petrarca nella costituzione del canone linguistico, rinvio a Trifone 2007: 19 e Tesi 2001: 71 .

${ }^{2}$ È però ugualmente riconosciuto che l'adesione del Bembo alla prassi linguistica petrarchesca non è né totale né tantomeno passiva, come ha dimostrato Carmelo Scavuzzo, fornendo numerosi esempi che attestato la divergenza fra la prassi poetica e la teoria grammaticale del cardinale veneziano (Scavuzzo: 181-207).

${ }^{3}$ Come ha modo di osservare Trovato, infatti: «Osservazioni diventerà un titolo frequente per opere che oggi diremmo di stilistica fondate sui modelli trecenteschi e che l'interesse grammaticale e linguistico per il Petrarca e le altre "corone" fiorentine era realmente più forte e precoce nelle corti settentrionali che in Toscana» (Trovato 1994: 82).

${ }^{4}$ Bembo rilanciava il concetto dell'indissolubilità dello stile di ogni scrittore, indicando Cicerone per la prosa e Virgilio per la poesia quali modelli incontrastati di perfezione stilistica e rifiutando l'imitazione eclettica, in un continuo sforzo di emulazione e superamento: "Totammihioporteteius stili facies exprimat, cuius se imitatorem divi vult, quemeo nomine dignumputem. [...] nostra demum contentio omnis id respiciat, ut quem assequuti fuerimus, etiam praetereamus». Al contrario, Pico della Mirandola aveva proposto l'imitazione di più modelli: «[...] imitari itaque eam debemus, quam animo scilicet gerimus dicendi perfectam facultatem, qua et aliorum et nostra cum errata in obeundo loquendi munere, tum virtutes etiam metiamur, sive ea ipsa
} 
maggior ragione, dunque, il ciceronismo del Bembo, afferma Giuseppe Patota, non è d'impronta teoretica ma sperimentale, poiché si adegua a un assunto fondamentale: l'ottimo modello letterario, scaturito dall'attenta osservazione dei classici volgari, è armonia, equilibrio, e, quindi, la grammatica che ne deriva è prima di tutto "grammatica dell'armonia» (Patota 1993: 107). Così, la pubblicazione delle Prose, oltre a rivitalizzare la "questione della lingua", sancì il trionfo della lirica di stretta osservanza petrarchesca, intesa come imitazione rigorosa di un solo modello, alla maniera dei latinisti cosiddetti ciceroniani ${ }^{5}$. Fin qui, nulla di nuovo. Eppure, se è risaputo che gran parte degli intellettuali dell'epoca intervennero con le loro riflessioni «spesso capziose, quasi sempre poco sistematiche» a questa disputa (Tesi 2001: 195), è meno noto, invece, che alcuni poeti trasferirono nei propri componimenti diversi punti del dibattito linguistico - divulgati comunemente sotto forma di trattati, dialoghi e libelli polemici -, non solo attraverso un uso trasgressivo della lingua letteraria nella prassi scrittoria, ma sollevando critiche esplicite, dal punto di vista teorico, al trionfo di una letteratura scleroticamente improntata al linguaggio petrarchesco. Mi riferisco a quello sparuto gruppo di autori meglio noti sotto l'etichetta di "satirico-burleschi", la cui produzione si concentra nel breve arco di tempo compreso fra il 1546 e il 1549: Ercole Bentivoglio con Le Satire et altre rime piacevoli, edite nel 1546 (Bentivoglio 1546); Pietro Nelli con i due libri de Le Satire alla Carlona, stampate fra il 1546 e il 1547 (Nelli 1546 e 1547); Gabriele Simeoni con Le Satire alla berniesca, pubblicate nel 1549 (Simeoni 1549), lo stesso anno in cui vedono la luce a Milano le Satire e capitoli piacevoli di Giovanni Agostino Caccia (Caccia 1549). L'ambiguità teorica di queste raccolte "miste" è del resto evidenziata dall'uso intercambiabile dei termini che indicano i due generi, come attestano i versi del novarese Caccia nella satira XVI (vv.7-9) della sua raccolta del 1549: «Io vorrei far stampar questi miei carmi / o satire, o capitoli giocosi / ch'essi si sian». Di certo dovette pesare sul giudizio, generalmente negativo, dei posteri la censura operata da Francesco Sansovino ${ }^{6}$, che, nel 1560, pubblicò il cospicuo volume dal titolo Sette libri di satire, nel quale fissava la contrapposizione fra lo «stile eroico e grave»e lo «stilumil e basso» della satira oraziana e ariostesca, senza fare alcun cenno al burlesco e a chi aveva mescolato le acque della satira con quelle della poesia giocosa, tanto da escludere del tutto dalla raccolta Caccia e Simeoni, mentre Nelli e Bentivoglio venivano pesantemente decurtati (per il ferrarese i sei primi capitoli, per il senese otto testi dal I libro). Solo recentemente gli studiosi hanno cercato di rivalutare questi autori, evidenziando gli elementi comuni di tali esperienze, condotte simultaneamente sul doppio versante satirico e burlesco: il metro, la predilezione per la forma epistolare in versi, l'opposizione alla poesia lirica di contenuto amoroso, ma soprattutto la loro

penitus innata sit idea, atque ab ipsa origine perfecta, sive tempore procedente multorum autorum lectione consumata» (Damianaki 2000: 625-629).

${ }^{5}$ Così Dionisotti ha sintetizzato tale importante snodo del pensiero bembiano: «L'uso del volgare guadagnava spazio. Le due lingue si allontanavano in un pacifico paragone delle vette da entrambe toccate, Virgilio e Cicerone nell'una, Petrarca e Boccaccio nell'altra» (Giovanardi 1998: 210).

${ }^{6}$ Una prova di questa scarsa fortuna è costituita dall'esiguità di edizioni moderne delle opere oggetto del nostro studio: a tutt'oggi sono state pubblicate solo le prime sei Satire del Bentivoglio (1987), quelle più «ariostesche», mentre sono stati esclusi i capitoli di ispirazione burlesca, lacuna in parte colmata dall'edizione della nona satira (Alla signora Agnola della lingua tosca) curata da Silvia Longhi nel volume Poeti del Cinquecento (1054-1060). Di Nelli Piero Floriani ha pubblicato, del primo libro del 1546, le satire I e XII, trascritte fedelmente, tranne che per le minime variazioni grafiche e i refusi (2000: 541-551). Più recente, è invece l'edizione integrale delle Satire e capitoli piacevoli di Giovanni Agostino Caccia (2013), da me allestita. 
contiguità cronologica - se si considera che i primi ternari del Berni sono quasi sovrapponibili alle Satire dell'Ariosto -, porrebbero l'accento sulla difficoltà di stabilire una netta distinzione fra i due generi (Longhi 1983: 229-31). C'è chi, come Martellini, si spinge a ipotizzare la nascita di uno strumento nuovo, un "supergenere", nel quale confluirebbero i caratteri peculiari dei due generi modello: coscienza della propria identità (pur nell'abbassamento del personaggio che dice "io") e censura dei vizi umani, polemica contro una poesia formata dalla passiva giustapposizione di formule fisse, uso di una lingua e di uno stile bassi, che si avvicinano il più possibile al parlato, e, infine, attualizzazione delle potenzialità della parola (Martellini 1989: 468). Sotto l'angolazione più specifica del dibattito linguistico, il periodo in cui sono pubblicate le loro opere è ancora dominato dalle soluzioni rigorosamente letterarie del Bembo: le affermazioni critiche e teoriche dei letterati fiorentini, volte ad affermare la prerogativa di intrinseca regolarità e dignità della lingua fiorentina dell'uso, non si sono ancora consolidate nel tentativo di conciliare la lezione bembiana con il fiorentinismo naturalistico, come avverrà con la proposta varchiana e quella salviatesca (Vitale 1996: 7-8). Un settore, dunque, degno di un supplemento d'indagine, mentre più conosciuti dei "satirico-burleschi" sono i poeti burleschi, come Berni e i suoi emuli, molti dei quali militarono prima nel sodalizio dei Vignaiuoli ${ }^{7}$ e, successivamente, nell'Accademia dei Virtuosi, la cui produzione rivela una programmatica avversione per una poesia eccessivamente rarefatta e ripetitiva: parodia, sistematica tensione anti-petrarchista ${ }^{8}$, sovvertimento dei tradizionali rapporti semantici del lessico poetico ${ }^{9}$, sono solo alcuni dei caratteri specifici di una tradizione, quella bernesca appunto, che ha suscitato nel corso degli anni notevole interesse da parte degli studiosi ${ }^{10}$. Pur essendo necessario distinguere gli usi spontanei da quelli dovuti a un consapevole abbassamento di registro, come ha giustamente osservato Luca Serianni $(2005 \text { : 3-7 })^{11}$, il codice burlesco è caratterizzato da una forte inclinazione realistica e mimetica alla rappresentazione del parlato, alla quale concorrono, ad esempio, la predilezione per le sfere semantiche correlate agli aspetti materiali della quotidianità, oppure il ricorso a locuzioni e usi fraseologici colloquiali legati all'oralità, il che evidenzia, una volta ancora, l'anima profondamente eccentrica del filone burlesco rispetto al tradizionale linguaggio

\footnotetext{
${ }^{7}$ La tradizionale denominazione di Accademia de' Vignaiuoli è, secondo Romei, «un'etichetta di comodo, convenzionale ed inesatta», trattandosi non di «un'associazione statuariamente definita», bensì di «una libera aggregazione di persone colte, unite da comuni consuetudini di vita e da condivisi interessi letterari» (Romei 2007: 209).

${ }^{8} \mathrm{Si}$ tratta di una presa di posizione già avvertita e condivisa dai contemporanei, come attestano le parole del Lasca, tratte dalla prefazione alla celeberrima giuntina del 1548: «avendo le petrarcherie, le squisitezze e le bemberie, anzi che no, mezzo ristucco e infastidito il mondo, perciocché ogni cosa è quasi piena di fiori, frondi, erbe, ombre, antri, quasi, aure soavi» (Opere burlesche 1760).

${ }^{9} \mathrm{Si}$ pensi, ad esempio, alla questione del linguaggio «segreto», che permetteva di leggere in chiave oscena tutti gli aspetti del reale, e che ha suscitato anche un certo dibattito fra gli studiosi, dai più «oltranzisti», come Jean Toscan, fino ai più prudenti nei confronti di una lettura «pansessuale» della poesia giocosa (Della Corte 2004).

${ }^{10}$ I due studi fondamentali sul capitolo bernesco sono di Silvia Longhi (1983) e di Danilo Romei (2007). L'unica indagine linguistica disponibile, condotta sui cinque capitoli di Giovanni Della Casa, è di Andrea Masini (1997). Annotazioni tematiche e linguistiche su Berni e berneschi si possono ricavare da Nigro (2002). Ricordiamo, infine, il volume curato da Paolo Orvieto e Lucia Brestolini (Orvieto/Brestolini 2000: 199-217).

${ }^{11}$ «È addirittura ovvio ricordare che ogni rappresentazione letteraria, anche quella più dichiaratamente naturalistica, è il risultato di un filtro che rielabora materiali di partenza, collocandoli in un organismo artificiale, comunque distante dalla realtà di partenza» (Serianni 2005: 3).
} 
poetico $^{12}$. In questo senso poesia bernesca e "satirico-burlesca" sembrano coincidere. Infatti, da una parte, gli autori burleschi danno spesso della propria scrittura una definizione di stile basso, umile (il Mauro, nel suo Capitolo in lode di Priapo, esordisce per l'appunto affermando: «Quell'io che già cantai con umil verso»), da cui nascono le immagini, riscontrabili presso il Mauro, del «lavoro di tela grossa» e del «panno ordito di canapo o di stoppa», e, nel Berni, dello «stile da muratori, insomma di una poesia dall'aria naturale, un po' selvatica e incolta, «avviluppata in rustiche vesti» (Longhi 1983: 214-215); dall'altra, Pietro Nelli non solo decide di intitolare la sua raccolta Satire alla carlona ${ }^{13}$, ma si presenta spesso come «uomo grosso» e «poeta da tre bagattini» (Martellini 1988: 468), definendo, inoltre, i suoi versi «versacci sgangherati» (Nelli 1546, sat. I, 64); e il Caccia, nella sat. XIII, 113 si definisce: «poeta dal dì da lavoro», ribadendo tale giudizio nella satira XVI (vv.7-9): «Poco bevvei del fonte d'Elicona, / però non è miracol, s'io ragiono, / come dice quel'altro, a la carlona» (V, 7$9)^{14}$, con un esplicito riferimento all'opera del suo illustre predecessore. Eppure, si badi bene, gran parte della poesia cosiddetta comico-realistica, pur richiamandosi per sua natura alla lingua colloquiale, resta una produzione colta: un'"ingenuità" tutta letteraria, insomma, come suggeriscono alcuni versi di Nelli («M'affatico in far credere alla gente / ch'io non mi v'affatichi, e che il mio verso / sia sempre in ponto fra la lingua e ' 1 dente», 1546, sat. I, 49-51), in cui si insiste appunto sulla natura segnatamente letteraria della propria operazione di abbassamento stilistico ${ }^{15}$.

\footnotetext{
${ }^{12}$ Nonostante la frammentarietà degli studi esistenti sul tema, bisogna pur sempre evidenziare una certa omogeneità e compattezza delle soluzioni linguistiche degli autori berneschi. Antonio Corsaro insiste sul fatto che, in questi poeti, la coscienza di appartenere a un sodalizio fatto di regole argomentative e linguistiche comuni finisce non di rado per prevalere sull'individualità poetica dei singoli autori (1997: 153), atteggiamento attestato dalla circolarità di opzioni stilistiche e linguistiche fra gli scrittori baioni, come ad esempio alcune catene rimiche (Della Casa, Mauro e Molza usano infatti la serie, di trafila ariostesca, vivande:ghiande:grande, cfr. Masini 1997: 184).

${ }_{13}$ Vale la pena soffermarsi su questa espressione, che riscuote un notevole successo presso Berni e i berneschi:usato sia come avverbio, con il valore di 'alla buona, con trascuratezza, in modo grossolano', oppure con valore di aggettivo, semplice, spensierato, grossolano', cfr. GDLI, II, s.v. carlona. La prima attestazione risale al Marescalco dell'Aretino (1533), cfr. DELI, I, s.v. alla carlona; si registra anche in Molza, Capitolo della scomunica, 13: «Parlando alla carlona io vuo' mostrare»; Berni, Capitolo del debito, 17: «voi chiamatela vita alla carlona»; Franzesi, Contra lo sberrettare, 33: «vivere a caso, e ire a la carlona»; e Della Casa, Capitolo del martello, 65: «E vivean sempre lieti a la carlona» (cfr. Masini 1997: 191 e 197); ma soprattutto Pietro Nelli, che intitola la sua raccolta satirica Le Satire alla carlona di Messer Andrea da Bergamo.

${ }^{14}$ Il che non vuol dire, come osserva Floriani, che l'autore operi una contaminazione dei codici per debole o erronea coscienza teorica: in questo caso, infatti, vuole solo insistere sulla modestia e l'umiltà (Floriani 1988: 175 e 184).

${ }^{15} \mathrm{Su}$ questo aspetto fondamentale della poesia giocosa, in quanto distanziamento dalla poesia "alta", rinvio a Orvieto; Brestolini 2000: 205-210.
} 


\section{Ercole Bentivoglio}

Ma al «primo proposito tornando» - per riprendere un'espressione del Berni - del presente studio, cioè individuare negli autori satirico-burleschi le istanze polemiche nei confronti dell'establishment linguistico e letterario dell'epoca, sarà utile ricordare che il processo di selezione operato dal Sansovino comportò - e mi domando fino a che punto si tratti di un'operazione casuale - l'omissione dei testi più critici nei confronti del fiorentinismo arcaizzante in auge all'epoca. È questo il caso del decimo capitolo delle Satire et altri capitoli piacevoli di Ercole Bentivoglio (1507-1573) ${ }^{16}$, intitolato $A$ la signora Agnola della lingua tosca, in gran parte dedicato alla questione linguistica ${ }^{17}$. Il capitolo è nettamente diviso in due parti: la prima, di 87 versi, ospita una pungente polemica contro le pretese del toscano, e particolarmente del canone riduttivo messo in opera dai petrarchisti; la seconda è una burlesca ed equivoca allocuzione alla signora Agnola $^{18}$, a cui si richiede il favore di istruire l'autore sull'uso del veneziano al posto del toscano, tutta giocata sul valore equivoco del lessico relativo all'insegnamento di una lingua ${ }^{19}$. La dedica della satira rinvia, dunque, alla ricca tematica "puttanesca" della poesia burlesca $^{20}$, che è di per sé una dichiarazione di dissenso rispetto alla poesia di

\footnotetext{
${ }^{16}$ Mantovano di nascita, fu legato alla corte estense e, a partire dalla seconda metà degli anni '40, all'ambiente della cultura di Venezia, città presso la quale si trasferì quasi definitivamente dal 1550. E proprio a Venezia, per i tipi del Giolito, escono nel 1546 le Satire et altri capitoli piacevoli: la satire sarebbero i primi sei capitoli, gli altri quattro dovrebbero essere classificati come rime piacevoli, accostandosi alla poesia burlesca. Seguendo questa suddivisione, Antonio Corsaro ha pubblicato, nel 1987, un'edizione critica delle prime sei satire. La divisione, accettata dal Sansovino nel 1560, non è del tutto soddisfacente: non è questa la sede più adatta per entrare nello spinoso dibattito sui tratti specifici della satira e della poesia burlesca, ma ricordo che dal punto di vista tematico e formale il discrimine fra satire e capitoli piacevoli non è sempre netto negli altri autori satirico-burleschi. Così, la lode del formaggio (settima satira) si inoltra nei territori del genere satirico, come l'elogio della vita rustica o la contrapposizione di comportamenti deprecabili a comportamenti saggi; il capitolo del Viaggio a Scandiano ripropone i resoconti berneschi di viaggi avventurosi e sgangherati, celebrando la tranquillità agreste. Il decimo e ultimo capitolo (A messer Antonio suo parente) ripropone motivi caricaturali cari alla poesia burlesca, ma con spunti riflessivi affini al registro satirico.

${ }^{17}$ Desumo il testo dall'edizione curata di Silvia Longhi 2001: 1054-1060, da cui traggo anche alcune annotazioni linguistiche.

${ }^{18}$ Secondo Floriani si tratterebbe della cortigiana Angela Del Moro (Floriani 1988: 130), meglio nota con il nome di Zaffetta, ricordata anche nella Tariffa delle puttane di Vinegia (Barzaghi 1980: 172-173) e nel capitolo di incerta attribuzione Gentil' mio Como, io mi partì da voi: «Ma voi almente l'avete scelte belle / che la delicata Angela del Moro / e la Flaminia son come dua stelle» (Terze rime 1539).

${ }^{19}$ Metafore sessuali grammaticali e scolastiche, attingendo simboli sessuali dagli oggetti del loro ambito (anche dalle lettere o dalla loro combinazione) erano diffuse sia nel mondo classico, greco e latino, sia nel periodo medievale, cfr. Adams 1996: 57-58. Nella poesia burlesca sono comuni questi riferimenti ambigui, basti ricordare il valore accordato al termine penna, ad esempio in Tansillo, il quale svilupperà questa metafora oscena nella satira VI a Simon Portio, 22-30: «Donne, che a farve i capei d'or sète use, / io pongo in mano a voi la penna mia [...] Pur ch'alcuna di voi meco si stia, / da cui la penna sia drizzata e retta [...] Da vostre man la penna mia si metta / nel calamaio e tirisi e, secondo / a voi piace, si meni o piano in fretta» (Tansillo 2010: 190).

${ }^{20}$ Sull'equivalenza fra signora e cortigiana, si ricordi che Alla sua signora, ugualmente meretrixhonesta, è dedicato il dialogo in terza rima La tariffa delle puttane di Venegia (Barzaghi 1980: 168), e che Berni dice di essere innamorato del saio regalatogli dal Bibbiena: «proprio come se fussi la signora» (Canzon d'un saio, 4). Giovanni Agostino Caccia, oltre a dedicare la satira XXI sulla «coda» alla Signora Ortensia - antroponimo classicheggiante suggestivamente cortigiano -, ribadisce, nella satira XII a Giovan Pietro Grazioso, che «Credete forse voi, se ben i' spendo/qualch'ora a starmi con qualche signora», in cui è evidente l'accezione del termine come «prostituta». Afferma Bazzetta che «Il Rinascimento denota come onorevole la donna che nell'età precedente si chiamava con nome infamante e nel 1500 la cortigiana è chiamata signora, ma l'uso
} 
contenuto amoroso tradizionale, come altri motivi denigratori della figura della donna del codice lirico petrarchista, legati al topos della caricatura delle bellezze muliebri, quale la descrizione delle "donne di montagna" o alla descrizione di amori "anomali", in particolare le passioni omosessuali (Longhi 2001: 230 e Orvieto; Brestolini 2000: 214217). Il capitolo esordisce con una risoluta difesa del latino, in un atteggiamento simile a quello assunto, al principio delle Prose del Bembo, del ferrarese Ercole Strozzi, fedele sostenitore dell'antica lingua e aristocratico spregiatore della lingua volgare, che schernisce «sì come vile e povera disonorata» (Bembo, Prose II, 1) ${ }^{21}$ : «D'esta lingua volgar, che tanto aborro, / Dico: Ahi misera, non fassi / più di te stima, e 'n basso loco or siedi, / soggetta a questa vil, che 'n alto stassi! / Vedi che fan gli anni volgendo, vedi / come va 'l mondo: ch'una Toscanella» (vv. 3-9). Su questo nodo teorico essenziale del dibattito linguistico contemporaneo, il Bentivoglio segue le posizioni dei teorici cortigiani più oltranzisti, come l'Equicola, il quale affermava che la conoscenza del latino - cioè la più ampia cognizione delle sue regole grammaticali, retoriche, stilistiche - rappresentava la griglia per scegliere i migliori autori della lingua volgare, vale a dire i più conservativi:

Non observo le regule del toscano se non tanto quanto al latino son conforme et le orecchie delectano, pero de et di non troverai senza lo articulo, Dio non Iddio, benché sequente vocale; in modo che dove li imitatori de la toscana lingua totalmente ogni studio poneno in lontanarsi dalla lingua latina, io ogni cura et diligentia ho usato approximarme ad quella (Drusi 1995: 57-58)

Un modello teorico condiviso da Castiglione ${ }^{22}$, il quale nel Cortegiano aveva affermato:

[...] né comprendo perché [...] la [lingua] toscana basta per nobilitare i vocaboli latini corrotti e manchi a dar loro tanta grazia che, così mutilati, ognun possa usarli per boni (il che non si nega), la lombarda o qualsivoglia altra non debba poter sostenere li medesimi latini puri, integri, proprii e non mutati in parte alcuna, tanto che siano tollerabili.(Drusi 1995: 58n)

Uguale atteggiamento assumerà l'Achillini, acceso sostenitore del plurilinguismo delle tre corone fiorentine, dopo aver elencato una lunga serie di latinismi presenti nelle loro opere, irridendo così il rifiuto del latino:

Se voi e questi moderni della thosca lingua imitatori fuggir al tutto il latino pur volete, il che Dante, petrarcha e '1 Boccaccio non hanno fuggito, [...] ma nelle poesie e prose loro assai l'hanno frequentato [...] meravigliomi, volendo al tutto nelli componimenti vostri tralasciarlo, che non vi deliberate li nomi, li caratteri o le imagini delle latine letere al tutto

\footnotetext{
degenerò e cortigiane furono chiamate tutte le donne pubbliche che sapevano valorizzarsi. Il nome di cortigiana non si confuse mai colla donna da bordello» (1930: 18).

${ }^{21}$ Sappiamo delle dispute di cortigiani estensi sul primato tra lingua toscana e ferrarese, che potevanpo anche degenerare in «alterchi e risse sanguinose», come accadde nel caso di un duello tra due gentiluomini nell'agosto 1530 (Vita di Ludovico Ariosto ricostruita su nuovi documenti, Ginevra, Olschki, 1930, p. 597.

${ }^{22}$ Eppure tocca proprio all'Equicola il merito di averlo condotto a criterio unico ed autonomo di elaborazione del volgare culto, cfr. Drusi 1995: 58.
} 
variare e formare delle nuove, acciò che dal latino assolutamente vi partiate.(Giovanardi 1998: 203)

Più avanti il Bentivoglio si rammarica di non aver imparato il greco, non avendo così potuto perfezionare la sua formazione umanistica, proprio come l'Ariosto nella VI satira (vv. 178-80: «che '1 saper ne la lingua de li Achei / non mi reputo onor, s'io non intendo / prima il parlar de li latini miei»). La constatazione dell'inarrestabile declino del latino a favore del volgare offre lo spunto per sostenere che la lingua tosca, privilegiata sopra gli altri dialetti, è ripetitiva e scialba, ingiustamente sopravvalutata, avviluppata da regole noiose, che conducono, quasi come logica conseguenza, al plagio, ricorrente nella poesia coeva, un motivo particolarmente caro, si vedrà in seguito, anche agli altri autori satirico-burleschi (vv. 10-27):

Ognun dice che questa è la più bella, ognun a questa età l'onora et ama, ognun l'adopra in scritti et in favella. [...]

Ch'è lingua tosca, altro ch'un mar aperto, ampio di ciancie, et un lascivo canto, con poco succo, e poca fama e merto? Un rubbar sempre quei c'han scritto tanto? Un far un bel miscuglio di parole, e d'epiteti adorno tutto quanto?

Del toscano si stigmatizza la ripetitività del lessico, cristallizzato in un repertorio di formule e stilemi, ampiamente diffusi dai petrarchisti (vv. 28-36):

Dir le rive odorate e le viole,

i ginestrevol' monti, limpide acque,

e dir: «O ben nate erbe, o chiaro sole»;

e un altresì, che sempre mi dispiacque, brutta parola, quantunque al Boccaccio ne le novelle sue di porla piacque?

Ch'è lingua tosca, altro che dir avaccio, e quinci e quindi, e lo congedo e chero, spagnuol, che mai nei versi miei non caccio?

Occorre inoltre sottolineare che il Bentivoglio, accanto all'inventario di topoipaessagistici largamente diffusi nella poesia petrarchista, stigmatizza l'inconsueto aggettivo ginestrevole, cioè 'pieni di ginestre', rifacendosi a un passo del II libro della prima redazione del 1505 degli Asolani, in cui appare l'espressione «ginestrevolemonticiuolo», poi cambiata, nell'edizione del 1530, in «lieta montagnetta» (Longhi 2001: 1056). Tale atteggiamento di avversione per termini letterari ormai ascrivibili a un repertorio trito e logoro era già evidente in chi propugnava la supremazia della lingua usata a corte, come Mario Equicola, censore di alcune voci considerate d'estrazione rustica (guari, altresi ed eglino), peraltro ricorrenti negli Asolani:

dove serò mio non troverai guari, altresì, eglino, non mala scriptione, oppennione, iddii, luoghi et simili, per ciò che como nel politico vivere, così nel parlare devemo in qualche parte dal ignorante vulgo essere diversi. Ché, quantunque così forsi il villan toscano pronuntie, noi da pastori ma da la citàdevemo il bel parlare eligere, né sequitare la vitiosa et 
corrupta consuetudine, della quale ne admonisce M. Tullio non usemo la prava regula; ne comanda debiamo polire et nectare ogni nostro parlare. (Drusi 1995: 62-63)

Così, Pierio Valeriano, nel suo Dialogo della volgar lingua ${ }^{23}$ fa pronunciare ad Antonio Marostica una suggestiva parodia dell'affettazione fiorentineggiante dei giovani nella Roma medicea di Leone X (Giovanardi 1998: 50):

Messer Angelo, non si può più vivere, dapoiché son usciti fuora certi soventi, certi eglino, certi uopi, certi chenti e simili strani galavroni: non posso passeggiar per Parione che vengono questi giovanotti dottarelli, barbette recitanti, e stanno ascoltando quel che ragioniamo insieme, e ci puntano negli accenti, nelle parole e nelle figure del dire, che non sono toscane, senza una compassion al mondo, ridendosi di noi, che, se ben avemo messo la barba bianca negli studi, non sapemo quello che mai non ci sognassemo d'imparare.

E accanto a ginestrevol il Bentivoglio registra altresì, avverbio tipicamente boccacciano, segnalato da Fortunio più che altro per l'esiguità delle sue attestazioni poetiche $^{24}$; avaccio, equivalente arcaico di 'tosto', voce considerata ormai in disuso da Bembo $^{25}$, ma non da Fortunio ${ }^{26}$; quinci e quindi, spesso appaiati in una dittologia ampiamente attestata sia nel Canzoniere (6 occorrenze) che nei Trionfi (3) e autorizzata sia dal Fortunio che dal cardinale veneziano ${ }^{27}$; e chero (Rvf CCXXXIV, 13), presentato come provenzalismo nelle Prose, al pari del già citato altresi ${ }^{28}$.

Il Bentivoglio prosegue poi la sua requisitoria contro il toscano elencando una serie di usi linguistici della koinè padana prima della codificazione bembiana (vv. 5560): «Lui per egli or si nota in versi e 'n prose, / li per le, lo per $i l$, un si per un se; / a ti per te si guarda, et altre cose. / Come Bernardo Tasso, che mi punse / le prose mie, perché su v'era absente: / "Lontanvuo' che diciate" mi soggiunse». Giova soffermarsi sulle opportune annotazioni linguistiche apportate dal poeta ferrarese in merito ad alcune deviazioni dalla norma letteraria, tutte ampiamente diffuse nella koinè cancelleresca e lirica quattro-cinquecentesca:

- «Lui per egli or si nota» (v. 55): sarebbe superfluo soffermarsi su un contrasto che ha pesato sulla tradizione grammaticale italiana per secoli ${ }^{29}$. Siffatta prescrizione,

\footnotetext{
${ }^{23}$ Per una ricostruzione delle vicende editoriali dell'opera, cfr. Drusi 1995: 27-28 e Giovanardi 1998: 50.

${ }^{24}$ Regole grammaticali, I, 237: «Altresí per similmente pose Dante nel canto XIX dell'Inferno, ove dice: La giù cascherò io altresí quando, et così lo pose nelle sue canzoni, et il Boccaccio in molti lochi; ma in niuno il Petrarca lo usò».

${ }^{25}$ Prose II, 21: «divenuta vile [...] per la sua vecchiezza».

${ }^{26}$ Regole grammaticali, I, 239: «Et avaccio usa la lingua tosca: Dante nel canto X: et io pregai il spirito più avaccio / che mi dicesse chi con lui si stava, et il verbo avacciare: Dante: che si avacciasser a divenir sante».

${ }^{27}$ Per il Fortunio, Regole grammaticali, I, 261: «Indi de loco, et quinci et quindi»; Prose, III, 57: «Sono Indi e Quindi, che quel medesimo portano, ciò è Di là e ancora Dapoi, e Quinci, Di qua e Da questo, e Linci, Di là, che a questa guisa medesima formò Dante. Dissersi eziandio Di quinci e Di quindi, che anco Di quivi alcuna volta si disse».

${ }^{28}$ Prose, I, 10: «Sí come è da credere che si pigliasse Chero, quantunque egli latina voce sia, essendo eziandio toscana voce Cerco, perciò che molto prima da' Provenzali fu questa voce ad usar presa, che da' Toscani; la qual poi torcendo, dissero Chereree Cherire, e Caendomolto anticamente, e Chesta. [...] È medesimamente Quadrello voce provenzale, e Onta e Prode e Talento e Tenzona e Gaio e Isnello e Guari e Sovente e Altresi $[\ldots] \gg$.

${ }_{29} \mathrm{Su}$ questo aspetto, che è stato a lungo uno dei cavalli di battaglia dell'insegnamento scolastico, rinvio a D'Achille 1990: 313-341 e Poggiogalli 1999: 117-123.
} 
nonostante costringesse $\mathrm{i}$ grammatici a formulare eccezioni alla regola per giustificare $\mathrm{i}$ casi in cui gli auctores violavano tale norma ${ }^{30}$, sembra non toccare le koinaiquattrocinquecentesche, né sul versante cancelleresco né tantomeno su quello lirico, in cui l'uso come nominativo di lui è lei è abbondantemente attestato (Vitale 1953: 88 e Id. 1992: 65).

- «Li per le» (v. 56): il valore di $l i$ (e gli) come dativo singolare femminile (invece di $l e$, cioè 'a lei'), e in genere qualsivoglia scambio delle due forme, non era ammesso né da Bembo né da Fortunio ${ }^{31}$, ma era invece diffuso nelle koinai cortigiane (Vitale 1992: $65)$.

- «Lo per $i l »(v .56)$ : l'autore può fare riferimento all'articolo masch. sing. o al pronome di $3^{\text {a }}$ pers. sing. Nel primo caso, il è forma segnatamente letteraria ${ }^{32}$ il cui uso è regolarizzato da $\mathrm{Bembo}^{33}$, che invece, nel secondo caso, non è precettivo, ma fa dipendere il ricorso all'una o all'altra forma da questioni di fonetica sintattica ${ }^{34}$. Le oscillazioni nelle scriptae di corte sono notevoli e corrispondono agli influssi esercitati dai modelli linguistici locali (Vitale 1953: 88 e Id. 1992: 65).

- «Un si per un se, / a ti per te» (vv. 56-57): ci troviamo dinnanzi a particelle pronominali collocabili sullo stesso piano, in quanto ipercorrettismi tipici della koinè padana, in cui erano diffuse le forme del tipo per ti, da mi, ecc. (Vitale 1953: 88 e Id. 1992: 65).

- absente (v. 59): si tratta di un latinismo semantico, il cui significato è 'lontano', usato non solo nella lirica da Vittoria Colonna e il Cariteo, ma anche nell'epica da Ariosto (Orlando furioso, XIX 4, 3). La prescrizione può essere motivata dalla sopravvivenza di uno dei tanti nessi latineggianti (in questo caso - $b s$-), fortemente osteggiati dai sostenitori del classicismo volgare ${ }^{35}$, primo fra tutti Giovan Francesco Fortunio, il quale aveva segnalato la questione ortografica come uno dei principali

\footnotetext{
${ }^{30}$ Tali giustificazioni possono essere riassunte in due fondamentali interpretazioni: lui e lei (e loro) possono essere usati come nominativo se hanno il valore di colui e colei; il valore come nominativo di lui e lei è ammesso qualora siano dipendenti dal verbo essere con il significato di 'contiene, ha in sé, dimostra', cfr. Poggiogalli 1999: 118-123.

${ }^{31}$ Prose, III, 19: «Con ciò sia cosa che in vece di $L u i$ s'è preso a dire $L i$, e $L e$ in vece di $L e i$ nel detto terzo caso, e $L o$ e $L a$ nel quarto altresí, nel numero del meno». Fortunio, in merito a due passi controversi della Commedia, afferma che (Regole grammaticali, I, 120-121): «Et se altrimenti si legge, come in alcun loco in ogni stampa si trova, devesi imputare allo errore del stampatore; come nel canto XXX dell'Inferno, parlando di Ecuba, si legge in alcuni testi: tanto dolor gli fé la mente torta [...] Et dove nel canto XIX del Purgatorio, parlandosi de' geomanti, si legge: surge per via che poco le sta bruna, è manifesto error medesimamente, attribuendosi lo pronome di femina a maschio»

${ }^{32}$ Infatti l'uso quattrocentesco fiorentino aveva generalizzato ele non $\mathrm{il}$, cfr. Patota 1996: LVII-LVIII.

${ }^{33}$ Prose, III, 9: «È l'articolo del maschio nel numero del meno, quando la voce, a cui esso si dà, incomincia da lettera che consonante sia, quello che voi diceste, $I l$; e quando da vocale, Lo; il quale nondimeno si vede alcuna volta usato eziandio dinanzi alle consonanti, e piú spesso da' piu antichi che da' meno».

${ }^{34}$ Prose, III, 19: «È oltre acciò che a queste voci, Il e $L a$ e $L o$, si leva loro bene spesso la vocale, quando hanno altre vocali innanzi o dopo la loro: $S^{\prime} i^{\prime}$ 'l dissi mai, in vece di dire Se io il dissi; e Amor l'inspiri, in vece di dire La inspiri; e $O$ chi l'affreni, in vece di dire Lo affreni». Solo nell'Ottocento l'uso di $i l$ è recepito come poetismo (serianni 2009: 175).

${ }^{35}$ La scelta operata da Bembo nel Petrarca aldino a favore dell'assimilazione dei nessi consonantici (fra questi anche $b s$ ) di contro a un uso alternante nell'originale si può infatti ascrivere a quella tendenza regolarizzatrice che è poi alla base della prassi imitativa cinquecentesca, atteggiamento riscontrabile anche nei commenti petrarcheschi, si vedrà in seguito. Sulle scelte ortografiche bembiane, cfr. Maraschio 1993: 175-177.
} 
motivi ispiratori delle sue Regole grammaticali, auspicando un riassestamento della grafia su basi non etimologiche ${ }^{36}$ :

Et quindi, la ortographia dalla latina assai diversa comprendendo, mi parve che, come li grammatici latini dalla osservatione degli approvati auttori loro latine regole hanno posto insieme, cosí nella volgar lingua, la quale invece di quella hoggidí usiamo communamente, con la osservationedellisopranomati tre auttori, in ciò degli altri primi, ad ogni studioso di lei il medesimo poter essere concesso».(Fortunio 2001: 4-5)

Di seguito il Bentivoglio allestisce una vera e propria rassegna di hàpax linguistici tratti dalla Commedia, per lo più dall'Inferno: si tratta di voci particolarmente espressive, arcaiche, coniazioni dantesche, parole d'altri dialetti o d'altre lingue), che occupa ben sedici versi (da 65 a 81): andi, ammoglia, avinghia, doglienza, adona, cinghia, Aleppe, chioccia, sipa, abbica, buia, ecc. ${ }^{37}$ La scelta di sottoporre a vaglio poetico il lessico di Dante non è, ovviamente casuale. Le controversie coeve sugli auctores possono essere sintetizzate, ha osservato Giovanardi (1998: 209), in questo modo: da una parte troviamo una linea sostanzialmente filopetrarchesca, impersonata dal Bembo, cui se ne oppone un'altra filodantesca capitanata dal Calmeta. In ogni caso, le dispute sono per lo più incentrate sul vocabolario accolto dai tre scrittori, all'interno del quale si cerca di separare, usando una metafora bembiana, «il grano dal loglio» ${ }^{38}$. ऐ̀ evidente, dunque, che il Bentivoglio si dichiara favorevole ad un ampliamento del ventaglio lessicale ammissibile in poesia, con l'assunzione di voci altrimenti escluse da un canone eccessivamente ristretto. Inoltre, il ferrarese vuole far riflettere sul tema della fisionomia linguistica dei dantismi, altra argomentazione tipicamente "cortigiana", per convincere il lettore della loro italianità, che è poi il miglior certificato di garanzia per chi è convinto che un'ipotesi di lingua nazionale non debba passare attraverso le forche caudine del modello tosco-fiorentino. Così, il Castellano, protagonista dell'omonimo dialogo trissiniano, confuta l'idea dell'italianità della lingua, evidenziando le parole non fiorentine usate da Dante e Petrarca, in base alla loro localizzazione ${ }^{39}$, come pure l'Achillini, convinto del plurilinguismo delle tre corone, le quali, a suo avviso, avrebbero usato per l'appunto una lingua comune, non il toscano. Alla fine della satira del Bentivoglio - dal v. 85 in poi -, si approda scherzosamente al veneziano: una scelta polemica e controcorrente messa in opera attraverso un esperimento di bilinguismo (vV. 118-126):

\footnotetext{
${ }^{36}$ In particolare, rispetto al nesso - $b s$-, il Fortunio dichiara che «quando $b$ nel latino è dinanci da $s$, si gemina, come assolto, [...] assentio» (Fortunio 2001: 180-181).

${ }^{37}$ E inoltre: compiange, approccia, burli, strupo, pulcro, viri, rovente, alleluia, lici, guaio, vivagno, $i^{\prime}$ dicerei, arca, grige, rattento, anima fuia, lurchi, burrato, loza, curro, èi, brollo, preco, ch'io tomi, forbi, stroscio, ripa discoscesa, berze, trei, gualdane, cennamella, a lo scoscio, di chiappa in chiappa, introcque, Tabernicch, accaffi, mette co, t'aia, raccoscio, l'abbo, soga, si trulla e Austericch.

${ }^{38}$ Prose, 2, XX: «molto spesso ora le latine voci, ora le straniere, che non sono state dalla Toscana ricevute, ora le vecchie del tutto e tralasciate, ora le non usate e rozze, ora le immonde e brutte, ora le durissime usando, e allo 'ncontro le pure e gentili alcuna volta mutando e guastando, e talora, senza alcuna scelta o regola, da sé formandone e fingendone, ha in maniera operato, che si può la sua Comediagiustamente rassomigliare ad un bello e spazioso campo di grano, che sia tutto d'avene e di logli e d'erbe sterili e dannose mescolato, o ad alcuna non potata vite al suo tempo, la quale si vede essere poscia la state sí di foglie e di pampini e di viticci ripiena, che se ne offendono le belle uve».

39 Sicilianismi (aggio e faraggio), lombardismi (poria e diria) e altre voci genericamente non fiorentine (molte, fra le quali, ricordiamo alma, quadrella e sovente), cfr. Pozzi 1988: 156-157.
} 
I' dirò 'nvece d'un bicchiero, un gotto,

invece d'una tavola, una tola;

seròdiscepol buon, starovvi sotto.

I' verrò dritto a quella vostra scola:

Dirò: «Vattene via, Tosco sbisao!»e non userò più tosca parola.

Tutti i miei versi finiranno in -AO;

e loderò la beltà vostra immensa,

le bionde trecce, e'l viso delicao,

che mi legâr, quando vi vidi 'n Sensa.

Ha osservato Silvia Longhi che la comparazione fra lingua fiorentina e veneziano è svolta con argomentazioni contrarie nelle Prose (I, 14-15) $)^{40}$ - in ossequio, probabilmente, all'approccio paradossale promosso dalla poesia burlesca -, laddove il Bembo spiega perché abbia composto gli Asolani «più tosto in lingua fiorentina [...] che in quella della città sua»: la scelta del vernacolo sarebbe dettata dall'amore, che dovrebbe essere naturale, per la propria lingua di provenienza, non da considerazioni di liceità formale e stilistica (Longhi 2001: 1059n). Le sperticate lodi del Bentivoglio nei confronti del veneziano, oltre a essere giustificate dal prestigio ormai raggiunto, nel Cinquecento, sia a livello politico che commerciale, da questa parlata, soprattutto nella sua variante internazionale, vale a dire il "veneziano coloniale" o "de là da mar", vanno interpretate, a mio avviso, considerando due importanti motivi della teoria cortigiana: da un canto, infatti, si è consapevoli dell'origine settentrionale, e in particolare veneta, di prestigiosi studiosi e letterati, che hanno dato lustro alla lingua del Petrarca assai più di quanto abbiano fatto i toscani (Giovanardi 1998: 198) ${ }^{41}$; dall'altro si sostiene, come abbiamo avuto modo di osservare precedentemente, che le tre Corone, in realtà, non scrissero in toscano ma in una lingua caratterizzata da un sostanziale plurilinguismo. La contiguità dei principi linguistici esposti dal Bentivoglio con alcune delle proposte dei teorici cortigiani sarebbe confermata dalla tesi esposta del Floriani, secondo il quale le satire bentivolesche rappresentano l'applicazione del codice satirico all'àmbito elitario e aristocratico della società cortigiana (Floriani 1988: 140): principi linguistici e ispirazione letteraria troverebbero, dunque, la loro perfetta espressione nella compagine della corte.

\footnotetext{
40 « [...] se voi per aventura seguitar quegli altri non voleste, i quali perciò che non sanno essi ragionar toscanamente, si fanno a credere che ben fatto sia quelli biasimare che cosí ragionano; per la qual cosa essi la costoro diligenza schernendo, senza legge alcuna scrivono, senza avertimento, e comunque gli porta la folle e vana licenza, che essi da sé s'hanno presa, cosí ne vanno ogni voce di qualunque popolo, ogni modo sciocco, ogni stemperata maniera di dire ne' loro ragionamenti portando, e in essi affermando che cosí si dee fare; o pure se voi al Bembo vi farete dire, perché è, che messer Pietro suo fratello i suoi Asolani libri piú tosto in lingua fiorentina dettati ha, che in quella della città sua -. Allora mio fratello, senza altro priego di messer Ercole aspettare, disse: - Hallo fatto per quella cagione, per la quale molti Greci, quantunque Ateniesi non fossero, pure piú volentieri i loro componimenti in lingua attica distendeano che in altra, sí come in quella che è nel vero piú vaga e piú gentile - .

${ }^{41}$ Nella replica del Castellano a Ercole Strozzi, protagonista dell'opera omonima del Trissino (1529), sono ricordati i nomi di Augurello, Bembo, Trifon Gabriele, Fortunio, Delfin, Fracastoro e Delminio, dai quali «vennero ne la nostra età le prime osservazioni e le prime regole de la lingua di lui [scil. del Petrarca]».
} 


\section{Pietro Nelli}

E dall'area padana spostiamoci alla toscanissima Siena, da cui proviene una delle voci più impetuose della poesia satirico-burlesca: Andrea da Bergamo, al secolo Pietro $\mathrm{Nelli}^{42}$. Di nuovo al centro di una satira è l'imitazione pedissequa e maldestra, legata, prima di tutto, all'uso di un lessico arcaico e stereotipico. Si tratta del capitolo Dipentura di se stesso a Lorenzo Venturi ${ }^{43}$, contenuto nel primo volume delle sue due monumentali raccolte (7v-8r; vv. 22-54):

Nel mio dir non si ascolta, e non si vede un quinci, o quindi, un'ancide, un altresi, un dirloti, un unquancho, un scinde, o fiede.

Mi piace usar vocaboli sanesi, non tirati con argani, o con ruote, perch'io vo' che i miei versi siano intesi.

Questi, ch'hanno oggimai lasciate vòte

le bisacce al Petrarca, e la scarsella.

e pieno '1 mondo d'uopi, e di carote.

Quasi mi fanno recer le budella

col parlar su lo stitico, e far mostra

come già il corvo dell'altrui gonnella;

non vuol forza, o sudor, la lingua nostra,

onde chiunche s'affanna in parlar fosco

averla in presto, e non di suo dimostra.

Oltre al richiamo al vuoto formulario della poesia petrarchesca, agli arcaismi, al plagio incombente insito nell'imitazione, tutti motivi già riscontrati nella satira bentivolesca, il Nelli rivendica un maggiore aperturismo della norma linguistica allorché fa riferimento ai "vocaboli sanesi», riprendendo le idee toscaniste del Tolomei $^{44}$ e anticipando, di qualche decennio, le proposte della "scuola senese",

\footnotetext{
${ }^{42}$ Nello stesso anno 1546, nella stessa Venezia, uscivano accanto alle satire del Bentivoglio, le Satire alla carlona di messer Andrea da Bergamo (16 satire), al secolo Pietro Nelli, seguite nel 1548 dal secondo libro (26 satire). Magrissimi i dati biografici di questo autore: si sa che nacque intorno al 1507 in territorio senese, da una coppia contadina, e fu 'adottato' quasi subito dal medico, 'lettore' nello Studio senese, Giustiniano Nelli, di cui era probabilmente figlio naturale. I suoi legami con la città di Siena sono testimoniati fino alla data della morte del padre (1541), e oltre; ma sua patria d'elezione dovette diventare Venezia, i cui ambienti e società sono ben documentati nella sua produzione satirica. Morì forse poco dopo la battaglia di Lepanto, come attestano alcuni suoi componimenti celebrativi della battaglia. Sulla sua biografia e sulla sua produzione rinvio a Corso 1954; Martellini 1989; Longhi 1988: 239-240; Floriani 1988: 138-161 e Id. 2000. Quest'ultimo ha sottolineato che uno dei tratti burleschi più evidenti delle satire nelliane è la presenza «dell'incontenibilità e dell'infrenabile bizzarria dell'ispirazione» usata per giustificare l'aggressività morale della sua «penna restia», amplificate dall'andamento apparentemente casuale e divagante (1988: 144 e 150-151).

${ }^{43}$ Dipentura, presentata come voce tipicamente senese dal Bargagli, per Serianni non è tuttavia estranea all'uso scritto e orale fiorentino, cfr. Bargagli 1976: 125n.

${ }^{44}$ Importante il ruolo da riformatore svolto dal Tolomei in àmbito linguistico; nel Polito (1525), sviluppando l'idea propria dei filotoscani che si dovesse «così scrivere come parlare e non parlare in un modo e in altro modo scrivere» in modo che «li scritti siano purissima imagine de le tue parole», indicava più volte Siena e la sua Accademia come il centro delle discussioni ortografiche (Vitale 1992b: 146), mentre nel Cesano (pubblicato nel 1555, ma composto all'epoca delle Prose del Bembo), sostenendo il rapporto genetico fra etrusco e toscano, conciliava la teoria dell'eredità regionale con la tradizionale teoria della trasformazione del latino ad opera dei barbari, a tutto vantaggio di un generico modello «toscano» della lingua (Marazzini 2000: 69-70). Le idee del Tolomei saranno accolte anche dal grammatico settentrionale Alessandro Citolini, il quale,
} 
sviluppatasi, fra Cinque e Seicento, grazie a letterati come Celso Cittadini, Orazio Lombardelli e Simone Bargagli (Bargagli 1976: IX e Marazzini 2000: 100-102). Parole «non tirate con argani, o con ruote» ${ }^{45}$ e che possano essere facilmente comprese dal pubblico: un'affermazione fortemente critica verso una lingua dominata esclusivamente dall'uso scritto e destinata a un pubblico d'élite, da ascrivere altresì all'interesse per la lingua parlata dimostrato dai fautori delle tesi cortigiana e toscanista. In questo senso il Nelli anticipa le idee del Bargagli, secondo il quale esiste una stretta connessione fra parlato e scritto, non tanto per esigenze di eleganza o di generico adeguamento ai tempi, quanto per l'obbligo di considerare come primi e naturali destinatari della scrittura i propri concittadini (Bargagli 1976: XXI). A ragione, dunque, Floriani ha evidenziato la natura profondamente cittadina e demotica della poesia nelliana, soprattutto per il «suo tentativo di trasferire il codice satirico dall'ambito elitario e aristocratico della società cortigiana all'ambito "popolare", al giro della società urbana» (Floriani 1988: 140), di cui difende, aggiungiamo noi, anche gli usi linguistici. Peraltro, uguale atteggiamento emerge nel Proemio alla Ficheide del Caro - membro dell'Accademia dei Virtuosi fondata dal Tolomei - in cui si esibisce un'opzione linguistica non arcaizzante: «Et quanto alla lingua, io mi protesto, che non voglio essere tenuto d'usare, né la Boccaccevole, né la Petrarchevole, ma solamente la pura, et pretta Toscana d'oggidì, et della comune quella parte, che anchora da essi Toscani è ricevuta» (Garavelli 2002: 61). Nelle dichiarazioni del poeta senese è possibile avvertire non solo un vivo e schietto ardore campanilistico, ma anche la linea teorica di chi si fa custode dei tesori idiomatici locali minacciati dall'accettazione indiscriminata del modello dominante (Bargagli 1976: XIX). Senza dimenticare che il Nelli lega la condanna della lingua imitativa di stampo bembiano a una dimensione etica, di ispirazione fortemente "satirica", cioè il richiamo al "vero" e alla lotta contro l'"adulazione" ${ }^{46}$. Il suo giudizio è ribadito nella satira XV Amicizia, a messer Benedetto:

Io scrivo com'io parlo tutto '1 giorno

senza sudar, senz'uopo, e senza unquanco

che non son di mia patria, né mai forno.

Usin le stitichezze i canta in banco

per parer petrarcheschi al volgo indotto,

che intende come lor, né più né manco.

E de sette di quei troverete otto,

che col suo scriver muffo altro non fanno,

che '1 predicar che fé '1 Piovano Arlotto.

Ma scriveno se pur tant'alto vanno,

del tolto impresto, io che sì basso dico,

non faccio agl'altrui libri furto o danno.

nella sua Lettera in difesa de la lingua volgare, sottolinea la natura viva della lingua volgare («ella è viva, e come viva cresce, genera, crea, produce, partorisce, e sempre si fa più ricca, e più abbondante»), riconoscendone la forma sostanzialmente toscana e non angustamente fiorentina e le piene funzioni, culturali e sociali, scritte e parlate, con le conseguenti implicazioni dei modi della oralità e di quelli della scrittura (Vitale 2006: 45-47).

${ }^{45}$ Ricordo che qui l'accenno alla «ruota» fa riferimento allo strumento di tortura, il che enfatizza l'idea di insopportabile costrizione sottesa al canone trecentesco.

${ }^{46}$ Tuttavia, come si è segnalato precedentemente, i versi del poeta, apparentemente «alla carlona», sono frutto di un intenso studio: in questo senso anche il Nelli si fa portavoce della necessità di osservare, proprio come il Bargagli del Turamino, «tutti i buoni ammaestramenti e tutte le migliori cautele statene donate da' maestri del ben parlare e del bene scrivere in ogni e ciascheduna parte» (Bargagli 1976: XXII). 
Nella sat. VIII Della rogna, a Diomede Nelli (34r-39v) si critica nuovamente la rogna petrarchesca e si riprendono i motivi analizzati precedentemente, attraverso la descrizione dello sterile processo creativo dei pedantuzzi che compongono versi per la loro fantesca ${ }^{47}$ :

Che direm della rogna petrarchesca, qual grattan con la ponta della penna quei ch'han la poesia per lor fantesca?

Questa ha molti e sì in sommo in la codenna che come l'uom la comincia a grattare, se qui si gratta, quivi uscire accenna. Ognun grattando pensa diventare marito delle Muse, ognun parente dell'Ariosto, e d'Apollo compare. Altri la gratta tirando col dente il pensier del Petrarca, come il drone tira ' 1 cuoio tagliato scarsamente.

Alcuni col dir dil mal delle persone, molti ancora col tòr l'altrui mantello, come l'asino già quel del leone.

così la sua postema, e 'l suo martello ciascun meglio che può grattar si sforza, giorno e notte stillandosi 'l cervello.

Veggio alcun che talor mitiga, e smorza questo suo poetico pizzicore, mostrando un sonettuzzo fatto a forza.

Il Nelli, come il Bentivoglio, segnala nei suoi versi alcune parole-chiave di una norma linguistica e letteraria obsolescente: uopo, voce invisa al Valeriano - lo si è visto precedentemente -, ma legittimata dal Bembo; unquanco, a cui dedica particolare attenzione il Fortunio, corredandola di esempi tratti dal Canzoniere (XXIX, 1-2 e LXXXII, 1) e dalla Commedia ( $P g$ IV, $76-77)^{48}$, a fronte di una menzione stringata da parte del Bembo ${ }^{49}$; mentre per quinci, quindi e altresi, iamdictum. La riprovazione di ancide (fiorentinismo di tradizione poetica, vanta 7 attestazioni nei $R v f)^{50}$, fiede (derivato dall'arcaico fiedere/fedire) ${ }^{51}$, scinde (1 occorrenza in $P g$ XI, 103 e 1 inTF II,

\footnotetext{
${ }^{47}$ È possibile che in questa denuncia degli amori ancillari sia implicita una critica nei confronti di Bentivoglio, il quale dedica gran parte della satira I (A Messer Andrea Napolitano) alla sua fantesca, attualizzando un motivo già presente nell'Orazio satirico (Serm. I, 1, 31-63); Giovanni Agostino Caccia, di cui ci occuperemo più avanti, riprende questo tema nella terza satira ( $A$ messer Bartolomeo Cazza suo parente) della sua raccolta, come personale soluzione al problema della vedovanza.

${ }^{48}$ Cfr. Fortunio 2001: 228-230.

${ }^{49}$ Prose III, 61: «È Unque, che si dice eziandio Unqua nel verso; et è $o$, che di queste due voci Unqua e Anco è composto, e vale quanto Ancor mai, e altro che al passato e alle rime non si dà, e con la particella, che niega, si pon sempre». Tale avverbio era stato invece stigmatizzato dal Berni, cfr. Al cardinale Ippolito de' Medici: «e metterò gli unquanco a mano e' guari»; e Capitolo a fra Bastian dal Piombo: "Ho visto qualche sua composizione: / son ignorante, e pur direi d'avélle / lette tutte nel mezzo di / Platone; / sì ch'egli è nuovo Apollo e nuovo Apelle: / tacete unquanco, pallide viole / e liquidi cristalli e fiere snelle: e' dice cose e voi dite parole».

${ }^{50}$ Sono infatti più numerose rispetto ai derivati dioccidere, cfr. Vitale 1996: 70-71.

51 Si tratta di un arcaismo che avrà parecchio successo nella tradizione poetica italiana: lo usa ancora D’Annunzio, come segnala Serianni (2001: 204 e 227).
} 
15) e dirloti(esemplificazione del tipo arcaico acc. + dat. $)^{52}$, tutti termini che trovano ugualmente spazio nella poesia lirica contemporanea, conferma l'insofferenza nei confronti di un codice linguistico riducibile alle forme di una grammatica senza vita né anima, fissata per sempre in un ristretto novero di pagine esemplari.

\section{L'area piemontese}

Al 1549 risalgono le altre due raccolte satirico-burlesche oggetto del nostro studio, e che riguardano l'area piemontese, la prima per il luogo di pubblicazione, Torino, la seconda per la provenienza dell'autore, Novara ${ }^{53}$. Nel capoluogo piemontese, infatti, escono, per i tipi di Martino Cravotto, le Satire alla berniesca di Gabriello Simeoni ${ }^{54}$, così presentate da Silvia Longhi: «nove capitoli [...] di fisionomia cangiante, con violenti scarti tematici che non basta a dissimulare l'uniforme piattezza di una lingua indotta e "comune"» (Longhi 1983: 240). E infatti, questa ricerca di una lingua "naturale", distante dalla pratica pedantesca del petrarchismo è messa al centro dell'opera del Simeoni, tanto da essere la protagonista della satira con cui esordisce la raccolta, intitolata Dello stile berniesco. La vera novità dell'intervento del Simeoni consiste nel puntare il dito contro un'altra categoria di autori, che, come osserva Garavelli (2002: 57), avevano letteralmente saturato il mercato librario primocinquecentesco, i commentatori di Dante e Petrarca, qui rappresentati da Cristoforo Landino e Alessandro Vellutello (I, 1-24):

Chi dice che 'l gentil compor berniesco non è il più bel che si leggesse mai, sta dell'ingegno e del giudizio fresco. Puossi con esso traer sospiri e guai, senza tanti uopi, quanchi, schivi e snelli, che dan che fare agli ignoranti assai. Voglion le feste, questi poverelli, passarsi il tempo con un libro in mano, senza tanti Landini, o Vellutelli; e giunti a un passo del Petrarca strano, o di quel diavol di Dante Alighieri, dicon leggendo che non è cristiano,

\footnotetext{
${ }^{52} \mathrm{La}$ sequenza acc. + dat., propria del fiorentino più antico e regredita nel corso del Trecento a favore del tipo dat. + acc., innovazione toscana occidentale, era piuttosto rara già nel Cinquecento, cfr. Maiden 1998: $22 \mathrm{e}$ 184-88; Rohlfs, II: 176; e Serianni 2001: 32, 115 e 161. Il primo tipo è saldamente attestato nel Boiardo lirico: su 15 casi di pronomi combinati registrati, Mengaldo ne segnala 5 del tipo arcaico (Mengaldo 1963: 158). Bembo, nelle Prose (III, 19), considera i due tipi equivalenti, anche se viene preferito, in quanto caratteristico del toscano, il tipo più arcaico: «Ma regola e legge che porre vi si possa, altra che il giudicio degli orecchi, io recare non vi saprei, se non questa: che il dire, Tal la mi trovo al petto, è propriamente uso della patria mia; là dove, Tal me la trovo, italiano sarebbe piú tosto che toscano, e in ogni modo meno di piacevolezza pare che abia in sé che il nostro, e per questo è egli per aventura men richiesto alle prose, le quali partire dalla naturale toscana usanza di poco si debbono -», cfr. Poggiogalli 1999: 144.

${ }^{53}$ Ricordo, comunque, che all'epoca Novara gravitava attorno al Ducato di Milano: entrerà a far parte del Piemonte solo nel Settecento.

${ }^{54}$ Poligrafo fiorentino, la cui attività si svolge fra Italia e Francia, dopo un breve periodo a Torino, si reca a Lione e a Parigi, dove intraprende la sua attività come collaboratore alle stampe di diversi editori. Muore nel 1574. Sulla sua biografia, certamente non avventurosa, rinvio a Floriani 1988: 176.
} 
così questi non hanno i lor piaceri, e quegl'altri scrivendo nel volgare manco hanno i premi del poeta interi. [...]

Se Dio il dicessi son pur cose ladre comporre in una lingua ch'è comune, e dir falangi, se tu puoi dir squadre.

La critica verso una lingua artificiosa e arcaizzante - gli uopi e i quanchi ${ }^{55}$ del v. 5 , insieme alle falangi del v. $24^{56}$-, rinvigorita dall'immagine del poetuzzo stravolto dinnanzi a un passo delle corone perché privo dell'opportuna glossa, ribadisce l'immagine di una poesia ridotta a mero repertorio di stilemi, priva d'ispirazione e noiosamente ripetitiva, a fronte dello stilberniesco, inteso genericamente quale libera espressione della personalità poetica e morale dell'autore (Floriani 1988: 181). Soffermarsi sull'esegesi petrarchesca nel Cinquecento comporta riflettere parallelamente su un dibattito alquanto complesso, la definizione del Canzoniere come "forma chiusa", cioè come armonica struttura di componimenti, o come semplice silloge di rime sparse. Mentre il Gesualdo preferiva rispettare la compagine vulgata, rifiutandosi di correggere Petrarca, il Vellutello optava per un completo sconvolgimento dell'architettura dei Fragmenta, distribuendo i testi in tre parti (in vita e in morte di Laura, e altri soggetti). Se diversi erano gli approcci all'opera, comune era invece il desiderio di restituire al Canzoniere una misura perfetta, per affermare così l'assoluta efficacia del modello, tanto da spingere gli esegeti a un eccesso di petrarchismo. In questo senso va inteso il processo di emendatio di Bernardino Daniello, discepolo prediletto di Trifon Gabriele, solerte correttore delle quartine asimmetriche del sonetto "irregolare" Non da l'Hispano a l'indo Ydaspe del Canzoniere (Rvf 210), di cui vuole eliminare l'hapax metrico, un «unicum difficile da accettare in un autore assurto a norma proprio per la serialità del suo dettato e del suo stile»: più bembista di Bembo, dunque, osserva Luca Marcozzi ${ }^{57}$. E se un lettore moderno è perfettamente consapevole che la poesia petrarchesca non può essere incardinata in alcuna norma generale, per un commentatore come il Daniello era fondamentale ridurre a norma e a unicità una serie di variazioni retoriche, tematiche e prosodiche (Marcozzi 2009: 146). Una reductio ad unum volta essenzialmente a favorire l'imitazione poetica, proponendo un modello normativo non solo per lingua e grammatica, ma anche per le questioni retoriche e metriche. Tuttavia, a monte di questi interventi, una constatazione: agli occhi dei cinquecenteschi fa difetto una strategia univoca di canzoniere inteso «come forma fissa e consolidata del discorso lirico» (Gorni 1993: 116-17). Ciò che interessava, infatti, era procurare una più facile leggibilità del testo, al fine di disporre il canzoniere come una vita in rime di Francesco Petrarca, per una sua più sua facile fruizione in chiave narrativa. Il commento portato alle estreme conseguenze, tanto da stravolgere il testo

\footnotetext{
${ }_{55}^{55}$ Per quanco, forma aferetica di unquanco, cfr. GDLI, XV, s.v. quanco.

56 La dittologia falangi e squadre si riscontra nell'Orlando furioso XVI, 23: «non dirò squadre, non dirò falange» e tre volte nell'Hercole di Giraldi Cinzio, X: «e ch'egli vinceria falange e squadre»; XIII: «in ordine porrà falange e squadre»; e XV: «le genti e insieme pon squadre e falange».

57 «[...] La distribuzione dei versi nelle due quartine è diseguale, con rime alternate nella prima quartina a fronte di uno schema a rime incrociate nella seconda. E dal momento che a Daniello non risulta che uno schema siffatto appaia altrove nel canzoniere, non gli resta altro da fare che correggerlo, credo per aderire all'idea che il Petrarca, modello normativo, non possa presentare punti oscuri o increspature», cfr. Marcozzi 2009: 146.
} 
originale, per rendere il canzoniere modello di poesia e di vita ${ }^{58}$ : un'opzione avvertita come insidiosa dagli accademici Virtuosi, ma anche dal Simeoni, proprio perché orientata a diffondere un'interpretazione del petrarchismo fortemente ideologica, platonizzante, giocata sulla confusione fra letteratura e vita (Garavelli 2002: 74-75). A questa visione totalizzante del dettato petrarchesco, ne viene opposta un'altra attualizzante nei confronti dei modelli, più ottimistica verso i moderni, in ogni caso critica verso la proposta del Bembo di ridurre la poesia a uno standard univoco. Una critica recisa contro i commentatori petrarcheschi e chi li seguiva alla lettera. Ma non vengono risparmiati neppure gli esegeti danteschi, qui rappresentati da Cristoforo Landino, autore del celeberrimo Comento alla Commedia, il cui approccio universalistico, di iniziazione all'intero patrimonio dottrinario antico e moderno, infastidiva molti intellettuali cinquecenteschi, insofferenti all'istituto stesso del commento, tanto da ripudiarne il nome sostituito con quello di esposizione o dichiarazione $^{59}$. Ma furono proprio enciclopedismo e funzionalità didattica a rendere l'opera del Landino viatico essenziale e insuperato a Dante, tanto da essere il commento dantesco a stampa più diffuso durante tutto il Cinquecento. Un magistero, tuttavia, che poco si adattava all'ideale di "poesia naturale" propugnato dal Simeoni, il cui atteggiamento è significativamente affine a quello dimostrato dagli accademici Virtuosi, autori di dicerie e paradossali glosse ai testi petrarcheschi, secondo i quali il Canzoniere è un testo già trasparente e comprensibile; e un commento che spiega ciò che è già trasparente è involontariamente la parodia di se stesso (Garavelli 2002: 74), come lo sprovveduto poetucolo privo dei commenti del Landino o del Vellutello. Non è quindi casuale che il Simeoni citi i Cicalamenti del Grappa $^{60}$, esposizione parodistica del sonetto Poi che mia speme è lunga a venir troppo (Rvf, LXXXVIII), in cui il Petrarca, parlando di Laura, canta le lodi e i benefici del malfrancese, a lui comunicato dall'amata (Lombardi 2002: 193-sgg.). Un monito agli eccessi di un'esegesi farraginosa e storicizzata, responsabile di aver formulato cavillose congetture e strampalati aneddoti (Garavelli 2002: 73), qui caricaturizzati grazie alla consueta formulazione paradossale burlesca (I, 25-27): «Di qui Petrarca avuta ha tanta fune, / ch'ha confessato ch'ebbe il mal francioso, / et d'amor buon le viscere digiune». Degna di nota l'espressione avere tanta fune che, come l'accenno alla corda del Nelli, mette in evidenza l'idea di forzatura e costrizione legate alla prassi esegetica e imitativa.

E nel Dialogo della volgar lingua di Pierio Valeriano il personaggio Tebaldeo interviene a dar man forte alle idee dell'altro interlocutore, Gian Giorgo Trissino, esprimendo una carica fortemente antitoscana (Giovanardi 1998: 143 e Drusi 1995: $28)^{61}$, a favore, invece, di una lingua meno connotata localmente, più "comune", insomma, come quella auspicata dal Simeoni («una lingua ch'è comune» ha modo di sottolineare al v. 23), in ogni caso libera da ceppi grammaticali e aperta agli apporti dell'uso. L'interesse per la questione normativa è quindi evidente anche in questo autore, ed è ulteriormente legato alla necessità di liberare le possibilità espressive

\footnotetext{
${ }^{58}$ Come sottolinea Trovato il «petrarchismo non fu un fenomeno puramente letterario ma di costume (si pensi ai tanti ritratti coevi di giovani trasognati con un'edizione tascabile del Petrarca in mano)», cfr. Trovato 1994: 123 .

${ }^{59}$ Per la definizione dei tratti caratteristici del Comento landiniano rinvio a Procaccioli 2001: 84-97.

${ }^{60}$ Il titolo completo dell'opera, pubblicata a Mantova nel 1545 per i tipi di Venturino Ruffinelli, è citato nella rubrica laterale.

${ }^{61}$ La princeps del Dialogo risale però al 1624, il che non toglie, ai fini della nostra indagine, che il Tebaldeo fosse considerat,o dal bellunese e dai contemporanei, un campione della poesia cortigiana.
} 
altrimenti inibite da una fedeltà pedantesca ai modelli. E se da parte del Simeoni è un «abbaglio critico», ha rilevato Floriani (1988: 180), assimilare il bernismo al petrarchismo prebembiano (impersonato dal Tebaldeo), è altrettanto opportuno cogliere in questa dichiarazione soprattutto una provocazione anticlassicista, o meglio, una denuncia nei confronti di una prassi imitativa totalizzante e univoca.

E veniamo all'ultima tappa di questo nostro itinerario, alle Satire et capitoli piacevoli del novarese Giovanni Agostino Caccia, pubblicate a Milano nel $1549^{62}$. Le satire offrono alcuni interessanti spunti di riflessione relativi al dibattito linguistico, soprattutto in merito al principio di imitazione, qui esemplificati mediante la descrizione di situazioni topiche della lirica petrarchista, e di poeti da strapazzo intenti a saccheggiare le «bisacce e le scarselle» del Petrarca, per usuare un'espressione coniata dal Nelli: in questo senso l'opera satirica del poeta novarese prosegue il processo di demistificazione del vuoto formulario petrarchesco, già iniziato nella prima raccolta poetica del 1546, le Rime. Esemplare, a questo proposito, la satira XII indirizzata all'amico Gian Pietro Grazioso, in cui l'autore confessa di avere ormai superato la passione amorosa ${ }^{63}$. Il disinganno e il ravvedimento dell'amante, ricollegabile non solo a referenti satirico-burleschi si esprime, più che in pronunciamenti teorici, segnatamente attraverso l'autoparodia (Stella Galbiati 1991: 97-101): dopo l'esibizione del vuoto formulario della lirica di contenuto amoroso, impostata non sul capovolgimento e sull'alterazione del modello (come accade di solito nei poeti burleschi), ma svelando la finzione del parlante nei suoi atteggiamenti di innamorato, il poeta fa il verso alla sua rimeria amorosa:

\footnotetext{
${ }^{62}$ Il volume non riporta il nome dell'editore, ma grazie al Sandal (1988: 16 e 108) sappiamo che fu Giovanni Antonio da Castiglione, famoso per aver introdotto nell'editoria milanese la stampa di opere in lingua spagnola. Per le notizie biografiche di questo poeta originario di Castellazzo Novarese, rinvio a Caccia 2010: 59-104.

${ }^{63}$ Questa consapevolezza e l'amaro ricordo che ne deriva, rinvia a precedenti classici, in particolar modo a Orazio: l'epodo 11 (5-6 «Hic tertiusDecember, ex quo destiti / Inachiafurere, silvis honorem decutit»), intessuto di topoi, come il contrasto fra amore e attività poetica (1-2 «Petti, nihil me sicutanteaiuvat/ scribereversiculos amore percussum gravi»), o i segni rivelatori dell'amore (8-11 «Conviviorum e paeniter / in quisamantem languor e silentium / arguit e latere petitus imo spiritus»); ma anche l'epodo 15, invettiva contro la donna che ha tradito il poeta, venendo meno a un giuramento, per un amante più ricco (il cui svolgimento tripartito è in parte ripreso in questa satira: rievocazione dell'idilio; invettiva contro l'amante; maledizione contro il rivale, cfr. Orazio 1991, II: 1000).
} 
Non nego già che, s'io seco ragiono, talor non dica: «I' vi son servitore, e l'alma, e l'l core, e la vita vi dono». Ma le parole non m'escon del core: anzi, vi fo saper che sempre fingo, ch'io non son, per Dio grazia, in tanto errore.

Ben è ver che sovente a una dipingo il mio amor, la mia doglia e la mia fede, se dentro ne le chiacchiere mi spingo. E dico: «Né 'l mar cinge, né '1 sol vede maggior amor del mio», et ella, sciocca, com'al Vangelo a quel ch'io dico crede. $\mathrm{Oh}$, che allegrezza l'alma e 'lcor le tocca, mentre si dà ad intender che sia vero ciò che mi sente uscir fuor de la bocca!Se mi sentiste quando mi dispero,

e quando poscia sto senza far motto, che l'un e l'altro faccio di leggiero, vipisciereste per le risa sotto, sentendo uscir da me tanti sospiri, chesembran ben ch'io sia da senno cotto.

Aidolor metto mano e ai martiri, a l'angoscia, a la pena, e a la doglia, e poscia talor sembra ch'io m'adiri, e ho di rider dentro sì gran voglia, che vi giuro ch'a pena mi ritegno, che 'n questa sciocca un tal error s' accoglia ... (XII, 10-36)

Il poeta novarese aveva già avuto modo di sottolineare la sua estraneità, almeno teorica, a una poesia di facile conio e pedissequamente imitativa, difendendosi apertamente dai maldicenti che lo accusavano di plagio (V, 2533):

E un di quei, forse, che pensando vanno, ogni sillaba a fatto, ogni parola, per morder quei ch'un opra fatto avranno, dirà talora: «Or ecco, questi invola quivi Petrarca, o Dante», s'avrò tolto dagli autor magni una dizion sola; poscia ' 1 maligno è sì ignorante, e stolto, che ne' suoi versi, s'egli n'avrà fatto, in mille error da tutti sarà colto.

Ma attenzione, questa insofferenza per l'imitazione passiva del modello petrarchesco non presuppone l'abbandono di un severo apprendistato umanistico: in tale senso vanno interpretate le richieste fatte a Marc'AntonioMaioragio, futuro precettore del figlio, nella satira VII, ispirata alla VI dell'Ariosto (VII, 130-138):

Ma sopra ' 1 tutto, fate ch'egli impari, poi che principio n'ha, greca dottrina, che fa gli uomini egregi al mondo rari, perch'ella è vera guida a la latina, sì come la latina a la toscana, che l'una senza l'altra mal camina. Fate ch'a la gran tromba mantovana, a l'Arpinate e al Venusino attenda, perché senza essi ogni fatica è vana.

Degno di nota, dunque, questo tentativo di definire il proprio rapporto con la poesia contemporanea, sempre più contesa e dilaniata fra imitatori attestati su un canone pedantescamente angusto - che il poeta stesso, del resto, aveva condiviso in gran parte della sua prima esperienza poetica, le Rime - e insofferenti riformatori, desiderosi di rivendicare la libertà assoluta dell'artista all'insegna del rinnovamento formale. Anche 
una dedica, infine, può indicare una precisa scelta linguistica, come quella della XVI satira, significativamente rivolta ai membri della senese Accademia degli Intronati, che conferma le simpatie del Caccia per un canone linguistico non arroccato su posizioni rigidamente fiorentiniste e arcaizzanti ${ }^{64}$.

\section{Conclusioni}

E allora, quali conclusioni possiamo trarre dall'analisi condotta sulla produzione satirico-burlesca degli anni '46-'49? Innanzi tutto mi preme sottolineare che l'infrazione degli autori satirico-burleschi e la sua carica innovativa non possono essere considerate delle semplici baie accademiche, né tantomeno definite genericamente come «manifestazioni antipetrarchiste», nel senso di rifiuto totale dell'esperienza lirica, perché proprio di tale codice è imbevuta e si nutre la loro poesia: l'idea di questi poeti come i «naturali nemici del petrarchismo» ${ }^{65}$ cozza contro la realtà, comune a molti di essi, di una doppia gestione, come Giovanni Agostino Caccia, autore di una corposa silloge lirica, le Rime del $1546^{66}$. Il programma linguistico propugnato da questi autori si può condensare in una serie di principi, che rinviano da vicino a proposte antibembiane sostenute dagli intellettuali dell'epoca, come l'idea di una «commune italica lingua ${ }^{67}$ o di un tollerante «toscanismo»: appoggio al latino e al greco come sostegno imprescindibile al toscano; coscienza della perfettibilità delle regole del volgare; rifiuto di un canone esclusivamente imperniato sugli autori del classicismo volgare; fedeltà a un ideale di lingua elegante, allo stesso tempo libera dai ceppi grammaticali e aperta agli apporti dell'uso; ostilità manifesta verso i fiorentinismi arcaizzanti. Significativo l'intervento plurilingue del Bentivoglio: un hapax che evidenzia - grazie alle modalità paradossali di ascendenza burlesca - il bisogno di fuga dal vocabolario della poesia tradizionale e dalla limitatezza necessitante del petrarchismo. A mio avviso, dunque, le dichiarazioni programmatiche di questi autori sono mirate a una medietà linguistica scevra, allo stesso tempo, da eccessi pedantescamente imitativi e pretenziosi - in sostanza gli stilemi petrarcheschi ridotti a elementi triti e consueti nel formulario poetico convenzionale - da una parte, ma anche dal pericolo di una totale mancanza di convenienza e di misura, nel rispetto, dunque, di una tradizione ormai consolidata, proprio perché, in questo modo, si vuole affermare che la propria esperienza di ibridazione satirico-burlesca è un'iniziativa legittima, non «un'espropriazione, indebita e anomala, dei territori della poesia» (Longhi 1983: 221). In questo modo si potevano controbattere le principali obiezioni rivolte, nelle Prose, dal Bembo al Calmeta, capro espiatorio della tesi cortigiana, accusata di essere un mero fantasma inafferrabile, perché intrinsecamente instabile e priva di una tradizione di

\footnotetext{
${ }^{64}$ L'Accademia degli Intronati fu infatti espressione della cosiddetta «scuola senese», contraddistinta dottrinariamente per la sostanziale convinzione del primato linguistico toscano, in avversione al fiorentinismo bembiano; per l'irrinunciabile fiducia nella vitalità della lingua parlata in perpetuo processo di miglioramento, pur nel rispetto degli auctores; per la convinzione del valore intrinseco della parlata senese nel quadro degli idiomi toscani e, quindi, della necessaria presenza nel canone degli scrittori autorevoli, degli autori senesi antichi e moderni (Vitale 1988: 143).

${ }^{65}$ L'espressione, coniata da Arturo Graf, è citata polemicamente da Silvia Longhi (1983: 219).

${ }^{66}$ Osserva infatti Corsaro che gli autori burleschi «sono intellettuali di formazione umanistica regolare e articolata, conoscono il latino e le letterature classiche, coltivano la poesia alta» (1997: 153): esemplare, in questo senso, il caso di Giovanni della Casa.

${ }^{67}$ La definizione è di Mario Equicola, cfr. Giovanardi 1996: 33.
} 
scrittura alle spalle (Giovanardi 1998: 39); biasimo del resto esteso al toscano contemporaneo, colpevole di riconoscere più dignità all'uso che all'esercizio letterario ${ }^{68}$. $\mathrm{Ci}$ si proponeva, in sostanza, di rifondare la lingua letteraria su un ideale plurivoco, aperto a influssi diversi, non cristallizzato entro gli schemi invalicabili di un sistema monolitico, rigidamente imitativo e autoreferenziale.

Per concludere, vorrei avvalermi di un'immagine tratta dal Commento di Ser Agresto $^{69}$, in cui il Berni è rappresentato come un ladro che ruba «la chiave del cancello» del giardino delle Muse, per coltivarvi, con «altri poeti baioni [...], chi le Pèsche, chi le Fave, chi i Citriuoli, chi i Carciofi, e chi d'altre sorti di frutta», quasi a sottolineare l'aspetto abusivo della poesia giocosa rispetto a un sistema letterario e linguistico incanalato su binari di ripetitivo decoro formale. Ebbene, credo che i poeti satirico-burleschi non avessero nessuna intenzione di rubare una chiave, bensì di costruire un'altra porta attraverso la quale entrare, a pieno diritto, nel giardino della poesia cinquecentesca.

\section{Bibliografia}

ADAMS, James Noel (1996):Il Vocabolario del sesso a Roma. Analisi del linguaggio sessuale nella latinità. Bari: Argo.

BARGAGLI, Scipione (1976): Il Turamino, ovvero del parlare e dello scriver sanese, a cura di Luca Serianni. Roma: Salerno.

BARZAGHI, Antonio (1980): Donne o cortigiane? La prostituzione a Venezia: documenti di costume dal XVI al XVIII secolo. Verona: Bertani.

BAZZETTA DE VEMENIA, Nino (1930): Venus imperatrix. Le cortigiane milanesi. Ricordi, aneddoti e documenti sulla vita galante di Milano e Lombardia dai Visconti al secolo XX. Novara: La Cisalpina.

BEMBO, Pietro (1978): Prose della volgar lingua. Torino: UTET.

BentivogLIO, Ercole (1546): Le Satire et altre rime piacevoli. Venezia: Giolito de' Ferrari.

- (1987): Satire, a cura di Antonio Corsaro. Ferrara: Deputazione Provinciale Ferrarese di Storia Patria.

CACCIA, Giovanni Agostino (1549): Le Satire, et altri capitoli piacevoli. Milano: [s.e.].

- (2013): Satire e capitoli piacevoli, a cura di Benedict Buono. Milano: Lampi di stampa.

Corsaro, Antonio (1997): "Giovanni Della Casa poeta comico". Per Giovanni Della Casa. Ricerche e contributi. Bologna: Cisalpino, 123-178.

Corso, Cosimo (1954): "Due scrittori senesi del Cinquecento (Pietro e Giustiniano Nelli)". Bullettino senese di storia patria. Vol. 61: 1-97 e 65-75.

D'ACHILLE, Paolo (1990): Sintassi del parlato e tradizione scritta della lingua italiana. Roma: Bonacci.

\footnotetext{
${ }^{68}$ Cfr. Prose, 1, XVI: «Perciò che, oltre che naturalmente suole avenire, che le cose delle quali abondiamo sono da noi men care avute, onde voi toschi, del vostro parlare meno abondevoli, meno stima ne fate che noi non facciamo, sì aviene egli ancora che, perciò che voi ci nascete e crescete, a voi pare di saperlo abastanza, per la qual cosa non ne cercate altramente gli scrittori, a quello del popolaresco uso tenendovi, senza passar più avanti, il quale nel vero non è mai così gentile, così vago, come sono le buone scritture».

${ }^{69}$ Questa favoletta allegorica, ricordata da Croce, viene ripresa da Longhi 1983: 220.
} 
DamianaKi, Chrysa (2000): "Liceità e pratica dell'imitazione delle Prose. Bembo e il recupero dell'antico nel primo Cinquecento", "Prose della volgar lingua" di Pietro Bembo. Milano: Istituto Editoriale Universitario Cisalpino, 617-654.

Della CORTE, Federico (2004): "Vent'anni dopo. Appunti in margine a Le carnavaldulanguage". Lingua e stile. Vol. 39/2: 227-48.

DRUSI, Riccardo (1995): La lingua "cortigiana romana". Note su un aspetto della questione cinquecentesca della lingua. Venezia: Il Cardo.

FLORIANI, Piero (1988): Il modello ariostesco. La satira classicistica nel Cinquecento. Roma: Bulzoni.

- (2000): "Ariosto come modello: il caso di Pietro Nelli", Fra satire e rime ariostesche.Milano: Istituto Editoriale Universitario Cisalpino, 529-551.

FORTUNIO, Giovan Francesco (2001): Regole grammaticali della volgar lingua. Padova: Antenore,

Garavelli, Enrico (2002): "Perché Prisciano non facci ceffo. Ser Agresto commentatore". Cumnotibusse et comentaribusse. L'esegesi parodistica e giocosa del Cinquecento. Vecchiarelli: Manziana, 57-77.

GIOVANARDI, Claudio (1998): La teoria cortigiana e il dibattito linguistico nel primo Cinquecento. Roma: Bulzoni.

LONGHI, Silvia (1983): Lusus. Il capitolo burlesco nel Cinquecento. Padova: Antenore.

- (2001): "Poeti burleschi, satirici e didascalici". Poeti del Cinquecento. Milano: Ricciardi, vol. I, 625-1175.

Mengaldo, Pier Vincenzo (1963): La lingua del Boiardo lirico, Firenze, Olschki.

MARASCHIO, Nicoletta (1993): "Grafia e ortografia: evoluzione e codificazione".Storia della Lingua italiana. Torino:Einaudi, vol. I, 139-227.

MARAZZINI, Claudio (2000): Da Dante alla lingua selvaggia: sette secoli di dibattiti sull'italiano. Roma: Carocci.

MARCOZZI, Luca (2009): "Varianti e normalizzazioni del testo nell'esegesi dei Rerum vulgariumfragmenta di Daniello, Muzio e Tassoni". La filologia dei testi d'autore. Firenze:Franco Cesati, 137-157.

MAsini, Andrea (1997): La lingua dei “capitoli”, in Per Giovanni Della Casa. Ricerche e contributi. Bologna: Cisalpino, 179-206.

Nelli, Pietro (1546): Le Satire alla carlona di messer Andrea da Bergamo. Venezia: Comin de Trino da Monferrato.

- (1548): Il secondo libro delle Satire alla carlona di messer Andrea da Bergamo. Venezia: Comin de Trino da Monferrato.

NigRO, Raffaele (2002): "Poeti burleschi del Cinquecento". Burchiello e burleschi. Roma: Istituto Poligrafico e Zecca dello Stato, 759-783.

OPERE BURLESCHE (1760): Il primo libro dell'opere burlesche. Usecht al Reno: Jacopo Broedelet.

PatotA, Giuseppe (1996): "Introduzione". Leon Battista Alberti. "Grammatichetta" e altri scritti sul volgare. Roma: Salerno, XI-LXXXIV.

—(1993): "I percorsi grammaticali". Storia della Lingua italiana. Torino:Einaudi, vol. I, 93-137.

Poggiogalli, Danilo (1999): La sintassi nella grammatiche del Cinquecento. Firenze: Accademia della Crusca.

PozzI, Mario (1988): Discussioni linguistiche del Cinquecento. Torino: UTET.

Procaccioli, Paolo (2001): "Introduzione". Cristoforo Landino. Comento sopra la Comedia. Roma: Salerno, vol. I, 9-105. 
ROMEI, Danilo (2007): "Da Leone X a Clemente VII". Scrittori toscani nella Roma dei papati medicei (1513-1534). Manziana: Vecchiarelli, 205-242.

SANDAL, Ennio (1988): L’arte della stampa a Milano nell'età di Carlo V. Notizie storiche e annali tipografici (1526-1556). Baden-Baden: ValentinKoerner.

SCAVuzzo, Carmelo (2000): "Le riserve bembiane sul Petrarca", "Prose della volgar lingua" di Pietro Bembo. Milano: Istituto Editoriale Universitario Cisalpino, 181207.

SERIANNI, Luca (2001): La lingua poetica italiana. Roma: Carocci.

SETTE LIBRI DI SATIRE (1560): Sette libri di satire di Lodovico Ariosto, HercoleBentivogli, Luigi Alamanni, Pietro Nelli, Antonio Vinciguerra e d'altri scrittori. Venezia: Francesco Sansovino.

SimeONI, Gabriello (1549): Le Satire alla berniesca. Torino: Martino Cravotto.

Stella Galbiati, Giuseppina (1991): Un poeta satirico del Cinquecento: Giovanni Agostino Caccia. Pisa: Giardini.

TANSILLO, Luigi (2010): Capitoli giocosi e satirici. L'Arca e L'Arco edizioni: Nola.

TERZE RIME (1539): Terze rime del Molza, del Varchi, del Dolce et d'altri. Venezia: Curzio Navò.

Trovato, Paolo (1994): Il primo Cinquecento. Bologna: Il Mulino.

TRIFONE, Pietro (2007): Malalingua. L'italiano scorretto da Dante a oggi. Bologna: Il Mulino.

Vitale, Maurizio (1988): La questione della lingua. Palermo: Palumbo.

-(1992a): "Il dialetto ingrediente intenzionale della poesia non toscana del secondo Quattrocento", Studi di storia della lingua italiana. Milano: LED, 49-94.

-(1992b): "La scuola «senese» nelle questioni linguistiche fra Cinque e Settecento", Studi di storia della lingua italiana. Milano: LED, 143-179.

- (1996):Il canone cruscante degli «auctores»e la lingua del «Canzoniere» del Petrarca, Firenze: Accademia della Crusca.

-(2006): "Senesismi in un grammatico settentrionale del Cinquecento". Divagazioni linguistiche dal Trecento al Novecento. Milano: Francesco Cesati, 45-51. 